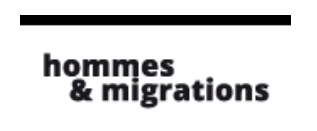

Hommes \& migrations

Revue française de référence sur les dynamiques

migratoires

$1326 \mid 2019$

Londres et ses migrations

\title{
Documenter les luttes de l'immigration. Regards croisés entre la France et l'Angleterre
}

Entretien avec Mogniss Abdallah, réalisateur et journaliste, et Ken Fero, réalisateur et chargé de cours en réalisation à la Regents University de Londres

\section{Anne Volery}

\section{(2) OpenEdition}

\section{Journals}

Édition électronique

URL : https://journals.openedition.org/hommesmigrations/10028

DOI : 10.4000/hommesmigrations. 10028

ISSN : 2262-3353

Éditeur

Musée national de l'histoire de l'immigration

Édition imprimée

Date de publication : 1 juillet 2019

Pagination : 158-167

ISBN : 978-2-919040-46-9

ISSN : $1142-852 X$

Référence électronique

Anne Volery, «Documenter les luttes de l'immigration. Regards croisés entre la France et

I'Angleterre », Hommes \& migrations [En ligne], 1326 | 2019, mis en ligne le 01 juillet 2019, consulté le

16 janvier 2022. URL : http://journals.openedition.org/hommesmigrations/10028 ; DOI : https://

doi.org/10.4000/hommesmigrations. 10028 


\title{
Documenter les luttes
}

\section{de l'immigration. Regards croisés entre la France et l'Angleterre}

\author{
Entretien avec Mogniss Abdallah \\ réalisateur et journaliste, \\ et Ken Fero, \\ réalisateur et chargé de cours en réalisation à la Regents University de Londres. \\ Propos recueillis par Anne Volery.
}

À l'occasion de l'exposition Paris-Londres, Music Migrations qui aborde notamment la question des crimes racistes et des violences policières dans les années 1980, nous avons rencontré Mogniss Abdallah et Ken Fero. Le premier est membre de l'agence IM'média (créée en 1983), une agence de presse basée en France et spécialisée sur l'immigration, les cultures urbaines et les mouvements sociaux, et le second membre de Migrant Media (créée en 1989), un collectif de cinéastes à Londres travaillant sur la production de documentaires autour des mêmes sujets. Les années 1980 ont été marquées en France et en Angleterre par une forte résurgence des crimes sécuritaires et des violences policières sur fond de crise économique, de chômage de masse et de racisme anti-immigrés. IM'média et Migrant Media sont nés de ce contexte particulièrement dur et d'une volonté commune d'enquêter sur ces crimes en les documentant, tout en inscrivant dans la longue durée les différentes formes de résistance politiques ou culturelles qui émergent de ces mobilisations. Retour sur leur travail et plus spécifiquement sur une série de films qu'ils ont réalisés et produits ensemble, Europe Communities of Resistance, exemple concret de regards croisés sur la situation dans les deux pays.

$H$

ommes \& Migrations : Vous êtes tous les deux réalisateurs et vous travaillez depuis les années 1980 sur les questions liées aux luttes de l'immigration et notamment sur la dénonciation des crimes racistes. Pouvez-vous revenir sur le contexte des années 1980 et sur votre travail, mais aussi sur votre rencontre et la décision de travailler ensemble ?

Ken Fero : À Londres, les années 1980 ont été très dures au niveau politique. Le thatchérisme, dirigé par la Première ministre Margaret Thatcher, dominait le Parti conservateur. Ce dernier était vraiment très à droite et les politiques publiques néolibérales qu'il mettait en place visaient directement les migrants, les réfugiés et les communautés noires.

On a eu, par exemple, les SUS Laws (Stopped Under Suspicion), les « lois d'arrestations sur simple suspicion », qui autorisaient la police à concentrer ses arrestations ou ses contrôles au faciès sur la jeunesse noire - généralement 
d'origine jamaïcaine et caribéenne. Ça a créé de la violence policière et des tensions. Aujourd'hui, on appellerait cela du « profilage racial ». Les années 1980 ont été marquées par une résistance massive face à ce phénomène : il y a eu des événements nationaux contre le racisme de la police, ainsi que des soulèvements en 1981 et en 1985. A contrario, il y a aussi eu de nombreuses agressions contre la communauté noire, comme le massacre de New Cross au cours duquel 13 adolescents noirs sont morts dans un incendie. Après cela, des organisations radicales se sont créé les unes après les autres dans les communautés noires.

Migrant Media s'est investi sur ces questions un peu plus tard, parce qu'on est arrivé après les communautés qui s'étaient installées suite à l'appel colonial vers les Caraïbes et l'Asie. On était très engagé dans les communautés noires, mais aussi dans les communautés du Moyen-Orient, d'Amérique latine ou d'autres pays. À l'époque, on décrivait Londres comme un melting pot, comme la ville de l'assimilation culturelle. Mais, en réalité, les communautés faisaient face à des événements violents à beaucoup d'égards et les gens se battaient sans cesse pour des droits basiques.

On faisait partie d'une sorte de mouvement politique militant et, pour nous, le militantisme ne se limitait pas simplement à documenter la vie quotidienne de nos communautés. Le militantisme, c'était documenter leur mémoire et leur résistance. C'est cela qui nous importait. Il ne s'agissait pas seulement de faire ou de montrer des films, mais bien de créer une archive de la mémoire puis de rassembler les générations, que ce soit dans les écoles ou à travers toute initiative non gouvernementale, afin de participer à l'éducation des gens.

On filmait sans cesse, on organisait des projections, des programmes d'éducation et des conférences et, quand on avait assez de contenu, on produisait nos films. On a cherché, sans succès, à savoir s'il y avait, au Royaume-Uni, d'autres personnes ou initiatives qui développaient un travail militant sur les médias et les communautés. Personne ne partageait notre position politique, aucun réalisateur ne travaillait de façon militante, en aidant les gens à s'organiser. Ils n'étaient pas prêts à faire partie du combat, ils se contentaient plutôt de filmer ou de projeter des images de ces luttes. Et puis on a entendu parler 
de l'agence IM'média en France et c'est comme ça qu'est née notre première collaboration autour de Britain's Black Legacy.

Mogniss Abdallah : Le contexte de la création de l'agence IM'média en France était différent. Avec la victoire de François Mitterrand à l'élection présidentielle (mai 1981), il y a eu une période de volontarisme politique sur la question de l'immigration au début des années 1980. Ce court instant a peut-être permis de changer la donne en termes de représentation, notamment celle des enfants de l'immigration. On n'était plus avec des immigrés qui étaient là seulement pour travailler, gagner leur croûte et puis repartir au bled. L'idée s'est installée que ces immigrés et leurs familles étaient là et qu'ils allaient rester.

Mais, très vite, est apparue une contradiction entre les déclarations d'intention et la réalité vécue par les immigrés et leurs descendants. De même, une partie de la société était très hostile à cette ouverture à l'immigration. Une des premières mesures du nouveau gouvernement socialiste avait été l'annulation des expulsions des enfants d'immigrés qui étaient monnaie courante jusqu'en 1981 et, suite à cela, il y a eu une contre-campagne très agressive. C'est par exemple ce qui a donné lieu à des affrontements avec la police aux Minguettes, dans la banlieue lyonnaise. À partir de cette période, la pression des crimes racistes et sécuritaires est redevenue très forte. Le mot d'ordre Pour le droit à la vie s'inscrivait dans l'opposition et la dénonciation des meurtres des personnes issues des différentes immigrations. IM'média, par ce biais, a développé un secteur d'enquête et de contre-enquête sur les violences de ce type et les réponses à leur apporter en termes de droit et de justice. C'est devenu de fait une spécialisation de l'agence.

Au milieu des années 1980, on a rencontré les camarades de Londres qui travaillaient sur ces mêmes questions. Des familles étaient organisées dans le cadre du Black Parents Movement par exemple, mais aussi des acteurs culturels et politiques de New Beacon et du collectif Race Today, avec des figures de proue comme John La Rose, Darcus Howe, Leïla Hassan ou Linton Kwesi Johnson (LKJ), connu en France pour sa « dub poetry ». Leur préoccupation était de savoir comment contrer les politiques de contrôle policier des quartiers populaires et de l'immigration. Comment s'organiser face aux violences policières?

LKJ avait un rapport à l'expression et à l'action publique qui nous correspondait bien. D'où l'idée de faire des allers-retours entre Paris et Londres de plus en plus fréquents. À partir de 1983-1984, c'était au moins deux fois par an. Au printemps, on allait à The International Book Fair of Radical Black and Third World Books, la foire internationale du livre radical noir et du tiers-monde. Elle se tenait dans différents quartiers à Londres, Leeds, Bradford, Manchester et Glasgow. Le deuxième rendez-vous annuel était le carnaval de Notting Hill. On aimait bien la musique, mais surtout l'idée que ce soient les habitants de ce quartier, à l'origine ouvrier et populaire, qui se le réapproprient pour un moment de fête. Cette idée a souvent été combattue par le pouvoir qui n'aimait pas trop que les gens organisent ce type d'événement réunissant plusieurs centaines de milliers de personnes hors du contrôle strict des autorités et de la police. 
Nous étions intéressés par le fait que c'était un événement autoproduit et par la mise en lumière d'une production culturelle particulière. Le carnaval de Notting Hill permet de revenir sur des idées communément admises en France, comme de penser que Londres c'est le multiculturalisme comme juxtaposition de cultures séparées les unes des autres. Nous, nous étions intéressés par la démarche qui consistait à articuler des expressions culturelles différentes dans un même espace.

K. F. : Nos premières collaborations avec l'agence nous ont beaucoup influencés. Par exemple, à cette époque, au Royaume-Uni et en particulier dans les quartiers est de Londres, il y avait beaucoup d'agressions à caractère racial contre les Asiatiques. Il y a eu de nombreux morts et, en réaction, l'organisation Rock Against Racism, basée sur l'idée que tout le monde pouvait vivre ensemble, qu'il ne fallait plus s'entre-tuer. C'était utopiste, d'autant qu'en réalité cette organisation n'avait aucun point d'ancrage dans la communauté. Il s'agissait simplement de sympathisants de gauche qui venaient pour dire : « Voilà comment 
résoudre le problème. »Cependant, en réalité, ils ne vivaient absolument pas la violence au jour le jour, ils ne faisaient pas face au racisme qu'il y avait là-bas, ni aux problèmes de classes. Il faut savoir que, dans ces quartiers, les conflits éclataient aussi entre la classe ouvrière blanche, les communautés asiatiques et l'extrême droite.

Rock Against Racism, c'était des bourgeois blancs libéraux qui disaient: « On peut régler le problème », mais, en réalité, ils n'ont rien réglé du tout. Et puis on est venu ici, en France, et on a entendu parler de Rock Against the Police. On a réalisé qu'il y avait une énorme différence entre les deux : en Grande-Bretagne, c'était Rock Against Racism et en France Rock Against the Police. Là, on pointait directement les personnes responsables du racisme dominant. Au RoyaumeUni, les gens voyaient Rock Against Racism comme un grand éveil culturel ou un acte de solidarité, alors qu'en réalité c'était assez pauvre politiquement. Rock Against Racism ne s'en prenait pas au réel problème de la police, or la police représente l'État, et la violence policière était omniprésente au RoyaumeUni. On a vu qu'en France, les gens se battaient contre ça.

Ce qui nous a aussi intéressés à notre arrivée en France, et au cours de notre rencontre avec Mogniss puis en travaillant avec l'agence, c'est cette idée de résistance qu'ils développaient. Ils documentaient avec des images une résistance à laquelle ils participaient eux-mêmes. On avait vraiment envie de prendre exemple sur eux parce qu'ils étaient en avance sur nous et qu'on voyait comment leur modèle de travail pouvait s'appliquer au Royaume-Uni.

H\&M : Vous avez débuté cette collaboration par une série de films sur ces problématiques intitulée Europe Communities of Resistance que vous avez co-réalisés et co-produits. La série comprend : Britain's Black Legacy, qui aborde la situation anglaise, Douce France, qui aborde celle de la France, et Germany, the Other Story qui parle de l'Allemagne. Comment est né ce projet ?

M. A. : On a vraiment commencé à travailler ensemble au moment de la chute du mur de Berlin. Il y a eu une forte montée du racisme, notamment dans cette Allemagne en pleine réunification. Des Noirs allemands s'étaient alors réfugiés à Londres. Ils se sentaient plus en sécurité dans le contexte multiethnique de la ville. L'idée d'Europe Communities of Resistance - en français Europe meurtres pour mémoire - est venue de nos multiples rencontres à la foire du livre ou encore au carnaval. La série a commencé avec deux films sur les violences racistes et policières en Allemagne (de l'Est et de l'Ouest).

Auparavant, l'agence IM'média avait fait des travaux avec d'autres acteurs et actrices en Angleterre. Notamment au début des années 1980, après les émeutes de Brixton, il y a eu le développement d'une classe moyenne noire qui s'est exprimée à travers des collectifs (Workshops Movements apparus en même temps que l'agence IM'média). Un certain nombre de militants, par exemple ceux de Race Today, ont basculé dans l'audiovisuel. Darcus Howe, Farrukh Dhondy et Linton Kwesi Johnson ont participé aux premières émissions multiculturelles de Channel 4, avec une dimension très militante. Licence to Kill a été une de nos premières collaborations internationales. Ce film, daté de 1985, est centré sur les meurtres racistes en France. Pour les Britanniques, il était 
inimaginable que des « beaufs 》 tirent au 22 long rifle, que des policiers puissent tuer et s'en sortir avec des peines dérisoires au mieux.

K. F. : Avec Communities of Resistance on voulait être assez provocateur et déclarer la notion de légitime défense comme partie intégrante de l'héritage noir. En Grande-Bretagne, il y a eu par exemple le cas des Bradford 12 : en 1981, douze jeunes d'origine asiatique avaient décidé de fabriquer des cocktails Molotov pour défendre leurs communautés parce que la police laissait l'extrême droite, le National Front, entrer dans leurs quartiers pour passer des gens à tabac. Ces jeunes ont donc fabriqué des cocktails Molotov et ils ont été découverts par la police, ce qui a mené à un grand procès à la Crown Court de Leeds (juridiction pénale de rang supérieur). Ils ont été jugés non coupables. Un jury entièrement composé de jurés blancs de la classe moyenne a jugé que ces douze jeunes d'origine asiatique, accusés de terrorisme, une accusation très grave, n'étaient pas coupables. Cette décision a envoyé un message très fort. Il y a eu beaucoup d'autres cas comme celui-ci, comme The Mangrove $9^{1}$ avec Darcus Howe, par exemple. C'est cette résistance et son histoire que nous voulions donner à voir, car la résistance a toujours existé, pour chaque personne tuée, une campagne est organisée. Un autre exemple : plusieurs femmes d'origine asiatique ont été licenciées de chez Grunwick ${ }^{2}$ parce qu'elles demandaient un salaire égal à celui des autres. Il y a eu une révolte. Ce sont des gens de la classe ouvrière, des personnes noires et asiatiques, qui ont mené ces combats. On tire de nombreux enseignements de tous ces cas, et ce encore aujourd'hui, 30 ou 40 ans plus tard.

1. En 1970, neuf militants noirs ont été jugés à la suite de violents affrontements avec la police lors d'une manifestation à Notting Hill. Le procès est devenu un procès politique lors duquel l'État a cherché à discréditer les dirigeants du mouvement, et notamment Darcus Howe. Après 55 jours passés à Old Bailey, les Mangrove Nine ont été acquittés et le racisme de la police a été mis en évidence.

2. En 1976, un groupe de travailleuses a entamé une grève à l'usine de Grunwick. C'étaient pour la plupart des immigrées d'Asie de l'Est qui revendiquaient un salaire égal et de meilleures conditions de travail. La grève, qui a duré deux ans, a donné lieu à plusieurs affrontements entre policiers et grévistes. Elle a également révélé les problèmes de sexisme sur les lieux de travail et de racisme dans les syndicats. 
On examine ces luttes pour voir ce qui s'est passé, comment les gens se sont organisés, parce qu'organiser une manifestation et une communauté demande énormément de travail et les médias ne s'y intéressaient pas trop. Ils venaient juste prendre des images et faire leur propre analyse, si on peut appeler ça comme ça. On avait vraiment une tout autre vision des choses. Communities of Resistance - Britain's Black Legacy parlait de ces 30 ans d'histoire de résistance face aux agressions racistes. Le film commence lors des toutes premières manifestations des années 1950 et il se termine avec la mort de Rolan Adams, en 1991, l'année où nous l'avons fini.

Douce France, quant à lui, a été un film crucial parce qu'il nous a appris des choses sur la situation en France. Il tentait notamment d'analyser comment la jeunesse réagissait face à la police ici. Il y était question des meurtres perpétrés par la police et de la situation dans laquelle nous nous trouvions en Grande-Bretagne. En fait, en Grande-Bretagne, les crimes de la police ont commencé depuis le jour où des gens d'ailleurs sont venus s'installer, et c'est précisément ce qui nous a motivés à faire le travail que nous faisons.

M. A. : Douce France est à l'origine un film de 52 minutes conçu pour Channel 4. Il a été diffusé sur cette chaîne qui, au début des années 1990, était encore en pointe sur les questions de représentation multiculturelle. Quand on a montré ce film avec la voix off de Linton Kwesi Johnson aux collègues d'IM'média en France, la réaction a été unanime. Les quinze années d'archives de l'agence sur l'histoire des luttes de l'immigration permettraient de faire quelque chose de plus exhaustif, plus particulièrement à destination du public français. La version longue, Douce France, la saga du mouvement beur a été très largement diffusé en France à partir de 1993.

L'intérêt de films comme Britain's Black Legacy ou Douce France est de montrer que les affaires dont ils traitent ne sont pas des cas isolés, elles sont à comprendre dans leur contexte et dans une continuité historique. Si on me demande de définir mon boulot, en tant que journaliste et réalisateur il me revient une formule qu'on avait à la création de l'agence : « On est des historiens de l'instant. » C'est dire que les acteurs et les actrices des situations doivent comprendre l'importance historique de ce qu'ils vivent et de ce qu'ils sont en train de faire. Ils sont capables de changer la donne. C'est le « making history » de Linton Kwesi Johnson.

H\&M : Vos films portent un fort engagement : vous documentez, vous créez une archive, mais vous créez également des liens entre les familles et vous les aidez à faire entendre leur voix dans l'optique d'une reconnaissance de leur combat. Vous pouvez revenir sur cet aspect de votre travail ?

K. F. : Réaliser Communities of Resistance et travailler avec l'agence nous a beaucoup appris et c'est devenu notre mode de travail jusqu'à aujourd'hui. Quand on regarde un film comme Injustice (Migrant Media, 2001), on y retrouve la même approche. D'abord, un travail de journalisme très rigoureux. On approfondit davantage notre travail d'investigation que la $\mathrm{BBC}$, davantage aussi que les journalistes qui écrivent pour le Guardian ou le Times, et le film 
Injustice en est la preuve. Au début du film, il est dit qu'en Grande-Bretagne, 1000 personnes ont été tuées par la police sur une période de 30 ans, entre 1969 et 1999. Avant de réaliser ce film, personne n'avait compté ces morts. Ils étaient traités comme des cas isolés. Maintenant, une organisation comme la BBC qui ne voit pas ce schéma, qui ne voit pas pourquoi cela arrive, pose un vrai problème. Or les gens ont été choqués par ce millier de morts. Ils nous disaient: " Je ne savais pas qu'il $Y$ en avait autant, je me souviens d'untel et d'untel... » Mais quand on se rendait compte du chiffre de 1000 morts, ça faisait un drôle d'effet.

Donc, la première chose est le travail d'enquête journalistique. Deuxième chose, il s'agissait d'aider les gens à s'organiser. Nous n'allions pas seulement quelque part pour filmer. Non, nous prenions vraiment part à l'organisation de manifestations et de meetings. Cela faisait partie du tournage, de la réalisation du film. Au moment où on discutait de ce qu'on allait filmer pour la fin d'Injustice, on s'est souvenu que, quelques années plus tôt, l'IRA avait organisé une procession entre Trafalgar Square et Downing Street où elle avait demandé à des membres du Black Panther Party de porter les cercueils de ses membres, ce qu'ils ont fait, symboliquement. Ce fut notre inspiration pour la fin du film et nous avons organisé une procession avec United Families \& Friends Campaign, la coalition des familles des morts en garde à vue. Voilà les messages que nous avons portés en nous basant sur l'histoire des luttes. Si on n'a pas connaissance de cette histoire des luttes, s'il n'y a pas de travail de mémoire, les mêmes choses se répéteront, encore et encore.

Le troisième élément, est la confrontation. Ce que j'entends par là, c'est qu'en montrant nos films on dévoile les visages des officiers de police, on cite leurs 
noms. En Grande-Bretagne, on n'est pas censé faire ça à cause des éventuelles poursuites judiciaires pour diffamation, mais on a des preuves. Des juges indépendants en ont pris connaissance et ils nous ont dit qu'effectivement: « Il s'agit de cas de meurtre ou d'homicide. » Donc s'il y a preuve, il faut procéder à une accusation.

La police a voulu éviter que le film soit montré en disant : "Vous ne pouvez pas dire ça, parce qu'on n'est pas coupables. » La question est de savoir si le risque de diffamation est plus important que le droit à la vie. Nous avons jugé que la question éthique est bien plus importante que la question légale, parce que le gouvernement britannique et les autorités se cachent derrière la question légale et ignorent la question éthique. Or si on n'a pas d'éthique, on n'a rien.

Je pense que cette méthode, basée sur un journalisme puissant, une organisation collective et la confrontation, est primordiale pour mener un travail collectif et pour que la jeunesse s'y intéresse. Il y a une histoire de la résistance, une histoire racontée par des réalisateurs qui ont fait des films très puissants depuis 30-40 ans. Est-ce qu'ils sont montrés à l'école ? Non. Dans les universités ? Non. Cette partie de l'histoire est ignorée et c'est pour ça qu'il est important que les gens la redécouvrent.

M. A. : Pour donner un exemple de l'inspiration commune entre Paris et Londres, on avait travaillé en 1984-1985 au rassemblement des mères des victimes de crimes racistes et sécuritaires, place Vendôme à Paris. Il y a eu plusieurs rassemblements qu'on a appelés « les Folles de la place Vendôme 》, ainsi que de nombreuses initiatives, toutes filmées et diffusées. Cela a été une source d'inspiration pour les familles en Angleterre. Injustice a été réalisé en 2001 . Il est présenté en France en 2019. Ce film donne à voir une dizaine de familles prises entre leur douleur et le besoin d'en parler, le besoin de se retrouver avec d'autres familles afin de rendre hommage aux victimes. L'enjeu est aussi de réclamer des réparations morales et, éventuellement, de faire aboutir les affaires aux niveaux national ou international. Le fait qu'il y ait des documents tels que ces films (Injustice, Les Folles de la place Vendôme), des archives télévisuelles, permet de faire vivre ces campagnes et de les transmettre aux nouvelles générations.

À écouter Ken, on a l'impression qu'à Londres, c'est encore plus fermé qu'en France. Je dirai plutôt qu'il y a des va-et-vient. À la fin des années 1980, IM'média a eu accès aux grands médias en coproduisant, par exemple, sur FR3 l'émission Rencontres, qui était comme une suite à Mosaïques. On avait pu parler des « Folles de la place Vendôme » à la télévision, on a pu écrire de nombreux papiers sur elles dans la presse écrite. Mais, peut-on dire que, sur le long terme, il y a plus de possibilités en France qu'en Angleterre ? La question reste entière.

K. F. : On a eu l'occasion de travailler pour la télévision, pour la BBC, au moment de la Guerre du Golfe avec After The Storm, ainsi que pour Channel 4 avec Douce France. Ça nous a fait prendre conscience du rôle de la télévision. En commençant notre enquête sur les morts perpétrées par la police, on a découvert que 
la télévision n'avait pas du tout envie de se confronter à ces sujets de cette manière-là. Ils ne voulaient pas y toucher.

En réalité, les producteurs multiculturels, qui faisaient plutôt un travail militant auparavant, ne se montraient plus si militants que ça. Quand on racontait l'histoire d'Injustice à des diffuseurs au cours d'un rendez-vous, ils nous répondaient : "Non, il n'Y a rien là, pas d'histoire. Est-ce que c'est vraiment arrivé d'ailleurs ? » Ils niaient la réalité de la situation. On a mis sept ans à réaliser ce film et ce, d'une part, parce qu'on n'avait aucun soutien et qu'on devait le financer nous-mêmes, et, d'autre part, parce qu'on voulait montrer comment les familles s'organisaient sur plusieurs années. On voulait montrer comment les gens changent. C'est vraiment central dans Injustice, on y voit comment les gens se sont investis dans la politique. C'était important de rassembler les familles dans ce film, à la façon des « Folles de la place Vendôme ».

La télévision anglaise a été choquée quand on a sorti le film et on a eu une approche stratégique pour cette sortie. On savait très bien que la police tuait des gens depuis 30 ans et que personne n'en parlait. La télévision parlait de violation des droits de l'homme en Chine et en Iran, alors que l'État au RoyaumeUni commettait des crimes sans qu'on en parle. Des personnes ont été tuées par la police, et ça c'est une violation des droits de l'homme. Personne n'avait jamais utilisé l'expression « violation des droits de l'homme » dans des cas comme ceux-là, mais on s'est inspiré du discours de Malcolm X devant les Nations unies qui a parlé de violation des droits de l'homme aux États-Unis - et non plus seulement de violation des droits civiques. On s'est dit que c'était la philosophie qu'il fallait suivre pour notre film et les combats des familles pour la justice.

Quand le film est sorti, la télévision a refusé de le montrer, la police a tenté d'empêcher sa diffusion et on a reçu des menaces de mort. Ils ont essayé de tuer le film, mais il est encore diffusé aujourd'hui, sans arrêt. Ils ne nous ont pas arrêtés. D'ailleurs, le message le plus important de ce film, c'est que la police ne peut pas toujours gagner. Si ce film n'existait pas, tout ce qui s'est passé et ces mille personnes tuées auraient été effacées de la mémoire nationale. C'est important de s'en rendre compte, parce qu'on ne sait pas de quoi sera fait l'avenir.

Une version filmée de cet entretien, comprenant des extraits des films Britain's Black Legacy et Douce France, la saga du mouvement beur, est visible en ligne sur le site Internet du Musée national de l'histoire de l'immigration (http://www.histoire-immigration.fr/paris-londres\#slide-6).

Plusieurs productions de l'agence IM'média sont visibles sur Youtube (https://www. youtube.com/user/agencelMmedia), et Migrant Media dispose d'une chaîne Viméo sur laquelle on peut notamment voir le film Injustice dans une version sous-titrée en français (https://vimeo.com/migrantmedia)

Traduction de l'anglais des réponses de Ken Fero par Marie Roth. 